\title{
Häufigkeit von pulmonalen Embolien und TBVT bei COVID-19-Patient/-innen
}

Suh YJ et al. Pulmonary Embolism and Deep Vein Thrombosis in COVID-19: A Systematic Review and Meta-Analysis. Radiology 2021; 298: E70-E80 doi:10.1148/ radiol.2020203557

Prothrombotische Gerinnungsanomalien und Thromboembolismus sind häufige Komplikationen bei schwer erkrankten COVID-19-Patient/-innen. Pulmonale Embolien (PE) sind die häufigsten thromboembolischen Ereignisse, die Assoziation zwischen PE und tiefen Venenthrombosen (TVT) bei COVID-19 ist bislang unklar. Ebenso unklar ist die diagnostische Genauigkeit von D-Dimer-Tests für PE. Beiden Fragestellungen widmet sich die Arbeitsgruppe mit einer Metaanalyse.

Su et al. führten eine Recherche in MEDLINE, Embase, Cochrane, MedRxiv und BioRxiv SSRN nach entsprechenden Veröffentlichungen von Januar bis Juni 2020 durch. Dabei suchten sie gezielt nach Studien zur Inzidenz von PE und Thromboembolismus inklusive TVT. Von 1646 identifizierten Studien wurden 27 mit 3342 COVID-19-Patient/-innen in die Analyse aufgenommen. 13 Studien mit 1896 Betroffenen berichteten über die Inzidenz von PE und TVT, 9 (1022 Betroffene) nur über die Inzidenz von PE und 5 (424 Betroffene) nur über die Inzidenz von TVT. Drei der 27 Studien waren prospektiv, die restlichen 24 retrospektiv. Nach der Newcastle-Ottawa-Skala waren 14 Studien von hoher und 13 von niedriger Qualität. Mit Ausnahme von 3 Studien erhielten alle Patient/-innen eine Antikoagulation in prophylaktischer oder therapeutischer Dosis. Suh et al. poolten die aus den Studien extrahierten Outcomes unter Anwendung des DerSimonian-LairdRandom-Effects-Modells. Eine weitere Evaluation erfolgte mit einer Meta-Regressionsanalyse. Primärer Outcome war die Inzidenz von PE und TVT, letztere begrenzt auf tiefe Beinvenenthrombosen (TBVT). Sekundäre Outcomes waren die Lokalisation der PEs sowie die diagnostische Genauigkeit von D-Dimer-Tests für PE bei COVID-19.

Für die PE betrugen die gepoolten Inzidenzraten 16,5\% (95\%-KI 11,6-22,9), für TBVT 14,8\% (95\%-KI 8,5-24,5). Eine Information über die PE-Lokalisation war in 318 Fällen aus 14 Studien verfügbar. Der größere Anteil war mit 64,4\% (95\%-KI 50,3$69,7)$ peripher lokalisiert, eine zentrale Lokalisation der PE war mit 39,0\% (95\%-KI 30,0-48,9) angegeben. In Studien mit Patient/-innen auf Intensivstationen oder schwerer Erkrankung wurden PEs mit 24,7\% (95\%-KI 1 8,6-32,1) häufiger angegeben als in Studien mit Nichtintensivpflichtigen. Hier betrug die angegebene Häufigkeit von PEs 10,5\%. Auch in Studien mit generellen pulmonalen CT-Angiografien bei allen Patient/-innen war die PE-Inzidenz höher. Eine gleichzeitige TBVT wurde bei 42,4\% der Fälle mit PE angegeben.

Elf Studien mit einer Studienpopulation von 567 Patient/-innen enthielten Angaben zu
D-Dimer-Konzentrationen und zu mit pulmonalen CT-Angiografien gesicherten PEs. Beim Vorliegen von PEs waren die D-DimerKonzentrationen mit einem Median von $7625 \mu \mathrm{g} / \mathrm{I}$ höher als in Fällen ohne PE mit einem Median von $1750 \mu \mathrm{g} / \mathrm{l}$. Die ROC-Kurve für D-Dimer-Tests wies für PEs eine AUC von 0,737 auf. Cut-off-Werte für D-DimerKonzentrationen von $500 \mu \mathrm{g} / \mathrm{l}$ und $1000 \mu \mathrm{g} /$ I hatten eine hohe Sensitivität für PEs von $96 \%$ und $91 \%$. Die Spezifität war mit entsprechenden $10 \%$ und $24 \%$ jedoch gering.

\section{FAZIT}

Su et al. fanden in ihrer Metaanalyse mit $16,5 \%$ und $14,8 \%$ hohe Inzidenzen von pulmonalen Embolien und TBVT. Bei Intensivpatient/-innen lag die PE-Inzidenz mit 24,7\% noch höher. In über $50 \%$ der Fälle mit PE bestand keine gleichzeitige TVBT. Die in vorbestehenden Richtlinien angegebenen CutOff-Werte für die D-Dimer-Konzentrationen zum Ausschluss von PEs erscheinen für COVID-19-Patient/-innen geeignet. Einschränkend sei die gezielte Suche nach Studien mit Thrombosen/Embolien zu berücksichtigen.

Gabriele Dobler, Berlin 